\title{
Size-dependent suppression of spin relaxation in electrostatic quantum dots
}

\author{
D. Chaney* and P. A. Maksym \\ Department of Physics and Astronomy, University of Leicester, Leicester LE1 7RH, United Kingdom
}

(Received 23 June 2006; revised manuscript received 7 November 2006; published 17 January 2007)

\begin{abstract}
Triplet-singlet spin relaxation in a vertical electrostatic quantum dot containing two interacting electrons is studied with a realistic model that includes the effects of finite thickness and screening. The spin relaxation mechanism is taken to be spin mixing via the part of the Dresselhaus Hamiltonian that is linear in the lateral momentum. The electron-electron interaction enhances relaxation and finite thickness suppresses it. The relaxation rate varies extremely rapidly with well width, $w$, approximately like $1 / w^{18}$ in zero magnetic field and $1 / w^{10}$ in a nonzero magnetic field. In addition, the rate oscillates with $w$ and this leads to further suppression of relaxation. If $w$ is sufficiently large the linear spin mixing mechanism is suppressed to the extent that it is no longer dominant.
\end{abstract}

DOI: $10.1103 /$ PhysRevB.75.035323

PACS number(s): 73.21.La, 72.25.Rb

\section{INTRODUCTION}

Spin relaxation in semiconductor quantum dots is currently under intense investigation because of its relevance to solid state quantum computing. Pioneering experiments by Fujisawa et al. ${ }^{1}$ have shown that the triplet-singlet relaxation rate in elliptical dots is very low, and this has been confirmed for other dot types and transitions. ${ }^{2,3}$ Theoretical work on spin flip processes was initiated by Khaetskii and Nazarov ${ }^{4}$ who studied excited state to ground state transitions in oneelectron circular and elliptical dots. They examined possible spin relaxation mechanisms and showed that linear spin mixing is the dominant one. Since then, excited to ground state transitions caused by spin mixing in various one-electron dot models have been studied theoretically ${ }^{5,6}$ as have transitions between Zeeman sublevels. ${ }^{7-9}$ However, most theoretical work so far has focused on idealized dot models, which do not include a realistic confinement potential, electronelectron interactions, and screening. The aim of the present work is to investigate these effects in a vertical pillar dot, the same class of device as used in the experiments of Fujisawa et al. ${ }^{1}$ As far as the authors are aware, there is only one other paper concerned with spin relaxation in a real device ${ }^{10}$ but this centers on a lateral dot which is very different from the vertical dot considered here.

The present work has been made possible by the development of an accurate device model in which confinement and screening parameters are obtained by fitting to experimental data. ${ }^{11,12}$ This leads to addition energies that agree with experimental data to around 5\%, so the model is considered to be a reliable description of the individual dot used to fit the model parameters and should also give reliable information about spin relaxation in the vertical class of device. However, the only device whose model parameters are known has circular symmetry while the device used by Fujisawa et al. has elliptical symmetry, so the present work is only applicable to the class of device used by Fujisawa et al. and not to the individual dot used by them. It would be possible to apply the present approach to the individual device used by Fujisawa $e t$ al. if it was characterized in the same way ${ }^{11}$ as the device considered here. The main finding of the present work is that the spin relaxation rate in the realistic model differs from rates calculated in idealized models by up to 4 orders of magnitude. In addition, the relaxation rate varies extremely rapidly with dot thickness, like $1 / w^{18}$ in zero magnetic field and $1 / w^{10}$ in a nonzero field. Consequently, a relatively small increase in dot thickness could suppress the linear spin mixing mechanism so that it is no longer dominant. This should make it possible to engineer a dot so that it has an extremely low triplet-singlet relaxation rate. However, relaxation in this regime would be dominated by effects such as the hyperfine interaction and coupling to the phonon strain field, which are thought to be small but have not yet been investigated in a realistic device model.

\section{THEORY}

The system considered here is an electrostatic pillar dot containing two interacting electrons. The dot is defined in a pillar which has heavily doped contacts at its top and bottom. The vertical confinement is provided by a finite depth, symmetric quantum well, of width $w$, and the lateral confinement is parabolic. ${ }^{13}$ There is a magnetic field, $B$, perpendicular to the plane of the dot. The electron wave function is taken to have the form $\chi\left(z_{1}\right) \chi\left(z_{2}\right) \Psi$, where $\chi$ is the vertical envelope function and $\Psi$ depends only on lateral and spin coordinates. $\Psi$ is found from an effective two-dimensional Hamiltonian, $H=H_{N I}+H_{I}+H_{S O} . H_{N I}$ consists of the kinetic energy, parabolic confinement potential, and Zeeman energy. The average effective mass, $m^{*}$ for the GaInAs-AlGaAs quantum well used to fabricate pillar dots ${ }^{13}$ ranges from $0.0673 m_{0}$ to $0.0648 m_{0}$ for well widths in the range $5-50 \mathrm{~nm}$. The parabolic confinement energy is $4.82 \mathrm{meV}$ and the effective $g$ factor, $\left|g^{*}\right|=0.3$. $H_{I}$ is the Coulomb interaction averaged over the vertical confinement and modified by the screening effect of the heavily doped contacts. The screening is found from the electrostatic Green's function ${ }^{12}$ and the screening length, $15.3 \mathrm{~nm}$, in the contacts is derived from experimental data. ${ }^{11}$ The dominant spin-orbit coupling mechanism in vertical pillar dots is the linear Dresselhaus mechanism. $H_{S O}$ therefore consists of the terms in the Dresselhaus spin-orbit coupling Hamiltonian that are linear in the lateral momentum, $H_{S O}=\gamma\left\langle p_{z}^{2}\right\rangle\left(\sigma_{y} \pi_{y}-\sigma_{x} \pi_{x}\right) / \hbar^{3}$, where $\boldsymbol{\pi}=\mathbf{p}_{\|}+e \mathbf{A}, \mathbf{p}$ is the momentum, $\mathbf{A}$ is the magnetic vector potential, $\left\langle p_{z}^{2}\right\rangle$ 
$=\left\langle\chi\left|p_{z}^{2}\right| \chi\right\rangle, \boldsymbol{\sigma}$ are the Pauli matrices and $\gamma$ is a material parameter ${ }^{14}$ whose value is in the range $26.2-27.5 \mathrm{eV} \AA^{3}$ for well widths in the range $5-50 \mathrm{~nm}$.

Although the linear Dresselhaus mechanism is the dominant source of spin-orbit coupling in pillar dots there are two other smaller effects, the cubic order Dresselhaus mechanism and the Rashba mechanism. The Rashba mechanism is important in systems with structural inversion asymmetry ${ }^{10,15-18}$ but the source of vertical confinement in the present system is an inversion symmetric quantum well. This is embedded in a pillar which stands on a substrate. This macroscopic device structure does not have inversion symmetry, but the dot is about an order of magnitude smaller than the pillar. Hence, symmetry breaking effects in the dot are expected to be weak. So, the Rashba coupling parameter for the present device is probably small but its actual value is not known. One effect of Rashba coupling is to change the ground state singlet-triplet transition from a crossing to an anticrossing. ${ }^{10,17}$ However, the main focus of the present work is on triplet-singlet relaxation and this occurs at fields below the singlet-triplet transition.

An important property of $H_{S O}$ is that it commutes with $L_{z}-S_{z}$, where $L_{z}$ and $S_{z}$, respectively, are the $z$ components of the total orbital and spin angular momentum. This is used extensively in the present work to block diagonalize $H$ and calculate rates accurately. The block diagonalization enables the eigenstates of $H$ to be labeled by the eigenvalues of $L_{z}-S_{z}$ and it is convenient to take these eigenvalues to be $-\hbar l_{s}$, where $l_{s}$ is a quantum number. The authors are not aware of this block diagonalization being used before, but it is needed to avoid systematic errors in numerically calculated rates for interacting systems.

Spin relaxation occurs when the eigenstates of $H$ are coupled by the electron-phonon interaction. Each state contains a small minority spin component and this results in transitions that are forbidden in the absence of spin-orbit coupling. The rate of transitions $R$ between state $\Psi_{i}$ and $\Psi_{f}$ is found from the Fermi golden rule and has the general form

$$
R=C\left(q_{0}\right)\left[N\left(q_{0}\right)+1\right] \int_{0}^{\pi} F_{\perp}^{2}\left(q_{0}, \theta\right) F_{\|}^{2}\left(q_{\|}\right) f(\theta) \sin \theta d \theta,
$$

where $F_{\|}\left(q_{\|}\right)=\left\langle\Psi_{f}\left|\Sigma_{k=1}^{2} \exp \left(-i \mathbf{q}_{\|} \cdot \mathbf{r}_{k}\right)\right| \Psi_{i}\right\rangle$ and $N$ is the Bose-Einstein factor (the temperature is $1 \mathrm{~K}$ ). Here $\mathbf{q}$ is the phonon wave vector, $\mathbf{r}$ is a vector in the plane of the dot, and $q_{0}=\Delta E / \hbar v_{s}$ where $\Delta E$ is the energy change in the transition and $v_{s}$ is the appropriate speed of sound. The functions $C, f$, and $F_{\perp}$ depend on the electron-phonon interaction. For the deformation potential interaction $C\left(q_{0}\right)=D^{2} q_{0}^{3} / 4 \pi \hbar \rho v_{s}^{2}, f(\theta)=1$, and $F_{\perp}=\left\langle\chi\left|\exp \left(-i q_{\perp} z\right)\right| \chi\right\rangle$. For the piezoelectric interaction $C\left(q_{0}\right)=\left(e h_{14}\right)^{2} q_{0} / 4 \pi \hbar \rho v_{s}^{2}$ and $f(\theta)=9 \sin ^{4} \theta \cos ^{2} \theta / 2$ for longitudinal phonons and $2 \sin ^{2} \theta\left[\cos ^{2} \theta+\left(3 \cos ^{2} \theta-1\right)^{2} / 4\right]$ for transverse phonons. The effects of screening and dielectric interfaces on the piezoelectric interaction are taken into account ${ }^{12}$ so $F_{\perp}$ $=q_{0}^{2} \iint \chi^{2}(z) G\left(q_{0} \sin \theta, z, z^{\prime}\right) \exp \left(-i q_{0} \cos \theta z^{\prime}\right) d z d z^{\prime}$, where $G$ is the electrostatic Green's function. The phonon modes are taken to be those of an elastic continuum. The material parameters for the $\mathrm{Ga}_{0.95} \mathrm{In}_{0.05} \mathrm{As}$ dot are: longitudinal $v_{s}$
$=4.69 \times 10^{3} \mathrm{~ms}^{-1}$, transverse $v_{s}=3.31 \times 10^{3} \mathrm{~ms}^{-1}$, density $\rho$ $=5.34 \times 10^{3} \mathrm{kgm}^{-3}$, deformation potential $D=8.49 \mathrm{eV}$, piezoelectric constant $h_{14}=0.154 \mathrm{~cm}^{-2}$, and dielectric constant $=12.8$. The barrier dielectric constant is 12.0. There is a wide range of $D$ and $h_{14}$ values in the literature, for example, $D$ for GaAs is $8.6 \mathrm{eV}$ in Ref. 19 and $5 \mathrm{eV}$ in Ref. 20. The values used here are the largest known to the authors so the calculated rates represent an upper limit.

The form factor $F_{\|}\left(q_{\|}\right)$is evaluated from numerically calculated eigenstates:

$$
F_{\|}\left(q_{\|}\right)=\sum_{\alpha \beta}\left\langle\Psi_{f}\left|c_{\alpha}^{\dagger} c_{\beta}\right| \Psi_{i}\right\rangle\left\langle\alpha\left|\exp \left(-i \mathbf{q}_{\|} \cdot \mathbf{r}\right)\right| \beta\right\rangle,
$$

where $|\alpha\rangle$ are the single electron states used to construct the two electron basis and $c_{\alpha}^{\dagger}$ and $c_{\beta}$ are electron creation and destruction operators. The form factor $F_{\perp}$ is found from $\chi(z)$. For a finite depth well $\chi(z)=A \cos (k z)$ when $|z|<w / 2$ and $\chi(z)=B \exp \left(-k^{\prime}|z|\right)$ when $|z| \geq w / 2$. Here $k^{2}=2 m_{w}^{*} E_{z} / \hbar^{2}$, $k^{\prime 2}=2 m_{b}^{*}\left(V_{0}-E_{z}\right) / \hbar^{2}, E_{z}$ is the well ground state energy measured from the bottom of the well, $V_{0}$ is the barrier height, and $m_{w}^{*}$ and $m_{b}^{*}$ are well and barrier masses, respectively. $k$ and $A / B$ are determined from the condition that $\chi$ and $\left(1 / m^{*}\right) d \chi / d z$ are continuous at $z= \pm w / 2$. This leads to the relation

$$
k \tan \frac{k w}{2}=\left(\frac{m_{w}^{*}}{m_{b}^{*}}\right)^{1 / 2}\left(k_{0}^{2}-k^{2}\right)^{1 / 2} .
$$

In the case of the deformation potential interaction, the form factor can be found analytically as $F_{\perp}=F_{\perp w}+F_{\perp b}$, where $F_{\perp w}$ and $F_{\perp b}$ are contributions from the well and barrier regions, respectively:

$$
\begin{aligned}
F_{\perp w}= & w A^{2}\left\{\frac{\sin \left[q_{\perp} w / 2\right]}{q_{\perp} w}+\frac{1}{2} \frac{\sin \left[\left(2 k-q_{\perp}\right) w / 2\right]}{\left(2 k-q_{\perp}\right) w}\right. \\
& \left.+\frac{1}{2} \frac{\sin \left[\left(2 k+q_{\perp}\right) w / 2\right]}{\left(2 k+q_{\perp}\right) w}\right\}, \\
F_{\perp b}= & w A^{2} \frac{\cos ^{2}[k w / 2]}{4 k^{\prime 2} w^{2}+q_{\perp}^{2} w^{2}}\left(4 k^{\prime} w \cos \left[q_{\perp} w / 2\right]\right. \\
& \left.-2 q_{\perp} w \sin \left[q_{\perp} w / 2\right]\right) .
\end{aligned}
$$

Here $q_{\perp}=q_{0} \cos \theta$ and $A$ is determined by the condition that $\chi$ is normalized to unity:

$$
w A^{2}=\frac{1}{\frac{1}{2}+\frac{\sin k w}{2 k w}+\frac{\cos ^{2}[k w / 2]}{k^{\prime} w}} .
$$

In the case of the screened piezoelectric interaction, $F_{\perp}$ is found from $\chi$ by numerically evaluating the Green's function integral stated in the previous paragraph. ${ }^{12}$

The electron-phonon matrix element for a single electron relaxing to the ground state in zero magnetic field vanishes ${ }^{4}$ to first and second order in $\gamma$. This result generalizes to triplet-singlet relaxation in a dot containing two interacting electrons; however, special precautions are needed to reproduce the perturbation theory results numerically. The eigenstates of $H_{N I}$ are the Fock-Darwin states, $|n, l\rangle$, where $n$ and 
$l$ are radial and angular momentum quantum numbers. The angular momentum factor as a function of the polar angle $\phi$ is $\exp (-i l \phi)$. The first order correction to $|n, l, \uparrow\rangle$ is -iar $\exp (-i \phi)|n, l, \downarrow\rangle$, where $a=m^{*} \gamma\left\langle p_{z}^{2}\right\rangle / \hbar^{4}$ and the first order correction to $|n, l, \downarrow\rangle$ is $-\operatorname{iar} \exp (i \phi)|n, l, \uparrow\rangle$. Thus, the angular momentum of the first order correction differs from the angular momentum of the unperturbed state by one unit. So if the eigenstates of $H$ are found by numerical diagonalization in a truncated Fock-Darwin basis, $H_{S O}$ couples some of the states in the basis to states outside the basis. Therefore, the numerical results are contaminated by systematic error, and this problem is severe when the electrons interact because $H_{I}$ mixes states with different single electron momenta and propagates the error from the "edges" of the basis to all the calculated eigenstates. This error is eliminated by using the eigenstates of $H_{N I}+H_{S O}$ as a basis for diagonalizing $H$. Then each basis state is correct to high order in $\gamma$ and the calculated rates are consistent with the results of perturbation theory. Typically the two-electron basis is constructed from one-electron states with $\left|l_{s}\right|<11 / 2$ and 12 one-electron states are used for each $l_{s}$ value. Then the Hamiltonian matrix dimension is typically a few hundred and the calculated rates are accurate to between $0.1 \%$ and a few percent, with the largest relative error in regions where the rates are both small and rapidly varying.

The eigenstates of $H_{N I}+H_{S O}$ are calculated by numerical diagonalization in the Fock-Darwin basis. The form of these states is constrained by the requirement that they are also eigenstates of $L_{z}-S_{z}$, and this enables all the necessary angular momentum states to be included in each basis state. The required states have the form $\left(\Sigma_{n} f_{n}|n, l\rangle\right)|\uparrow\rangle$ $+i\left(\sum_{n} g_{n}|n, l+1\rangle\right)|\downarrow\rangle$, where $f_{n}$ and $g_{n}$ are real coefficients. When $l_{s}>0$, writing the coefficient vector in the form $\left(f_{0}, g_{0}, f_{1}, g_{1}, \ldots\right)$ leads to a real tridiagonal eigenvalue problem. For $l_{s}<0$ the order $\left(g_{0}, f_{0}, g_{1}, f_{1}, \ldots\right)$ leads to a similar problem. The convergence is rapid and only states with $n$ $\leq 6$ are needed.

\section{RESULTS}

The low-lying energy levels of the interacting twoelectron system with well width $12 \mathrm{~nm}$ are shown in the top frame of Fig. 1 as a function of $B$. Below the singlet-triplet transition $(B \simeq 3.8 \mathrm{~T})$ the ground state has $l_{s}=0$ and the first excited state has $l_{s}=2$. The transition studied here (arrow) is between the lowest $l_{s}=2$ level and the lowest $l_{s}=0$ level and corresponds to triplet-singlet relaxation when the field is $\$ 3.8 \mathrm{~T}$. The region of the singlet-triplet transition is shown in the inset to the top frame of Fig. 1. Three Zeeman split levels lie close together in energy. There is a second crossing near 3.9 T with an anticrossing near $4.05 \mathrm{~T}$. In the presence of Rashba coupling the crossing at the singlet-triplet transition would become an anticrossing. ${ }^{10,17}$

The rates of triplet-singlet relaxation via the deformation potential interaction are shown in the bottom frame of Fig. 1 as a function of $w$. The rates for $B=0 \mathrm{~T}$ and $B=1 \mathrm{~T}$ oscillate and decrease rapidly with $w$ but the rate for $B=0 \mathrm{~T}$ is smaller and decreases faster than the rate for $B=1 \mathrm{~T}$. The rapid de-
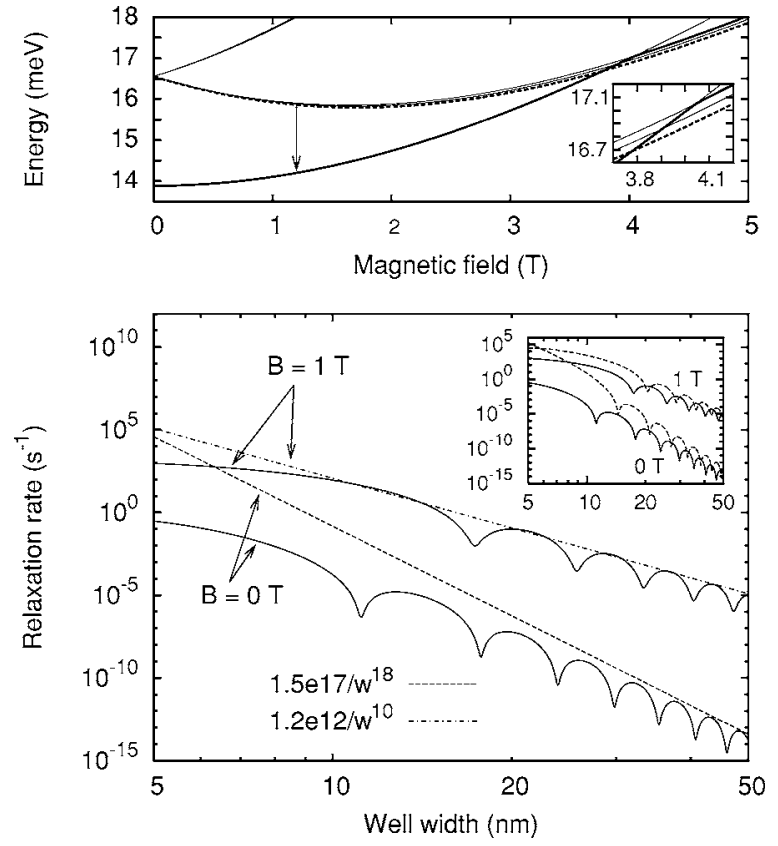

FIG. 1. Upper frame: low lying energy levels of a two-electron dot. Solid bold line: lowest $l_{s}=0$ level; dashed bold line: lowest $l_{s}=2$ level; arrow: transition considered in this work; inset: region near the singlet-triplet transition. Lower frame: Spin relaxation rates and theoretical asymptotic forms. Inset: rates for finite (solid lines) and infinite (dashed lines) well.

crease can be understood by considering the regime of large $w$. The solution to Eq. (3) in this regime has the form $k w$ $\approx \pi(1+\varepsilon)$, where $\varepsilon$ is $O(1 / w)$. This approximation is valid provided $E_{z} \ll V_{0}$ and allows $F_{\perp}$ to be approximated provided that $\varepsilon q_{\perp} w / 2 \ll \pi \sqrt{2}$; both conditions are satisfied for the parameter values considered here. The resulting approximation is

$$
F_{\perp} \approx(1+\varepsilon) \frac{\sin \left[q_{\perp} w(1-\varepsilon)\right] / 2}{q_{\perp} w / 2} \frac{\pi^{2}}{\left(\pi^{2}-q_{\perp}^{2} w^{2} / 4\right)},
$$

which coincides with the form factor for an infinite barrier well when $\varepsilon$ is set to zero. $F_{\perp}$ clearly oscillates with $w$ and decreases like $1 / w^{3}$. So $F_{\perp}^{2}$ decreases with a $1 / w^{6}$ envelope. The spin-orbit coupling parameter $\gamma\left\langle p_{z}^{2}\right\rangle$ decreases like $1 / w^{2}$ in the large $w$ regime. For $B=0 \mathrm{~T}$, the first and second order contributions to $F_{\|}$vanish. Therefore $F_{\|} \propto\left(\gamma\left\langle p_{z}^{2}\right\rangle\right)^{3} \propto 1 / w^{6}$, $F_{\|}^{2} \propto 1 / w^{12}$, and the rate decreases like $1 / w^{18}$ in the large $w$ regime. Figure 1 shows that the numerical results for $B=0$ are consistent with the asymptotic scaling when $w \geq 45 \mathrm{~nm}$. For $B=1 \mathrm{~T}$, the first order contribution to $F_{\|}$does not vanish and the asymptotic scaling is $1 / w^{10}$, consistent with Fig. 1. A decrease of rate with $w$ for single electron relaxation from Zeeman sublevels in an infinite barrier dot is reported in Ref. 9. However, the corresponding $w$ scaling law is not discussed so it is difficult to compare this case with the present results.

The rate oscillations are caused by zeros in $F_{\perp}$. The association between rate minima and zeros of $F_{\perp}$ has already been made. ${ }^{9,19}$ However, $F_{\perp}$ appears under an integral and, as far as the authors are aware, no explanation has been 


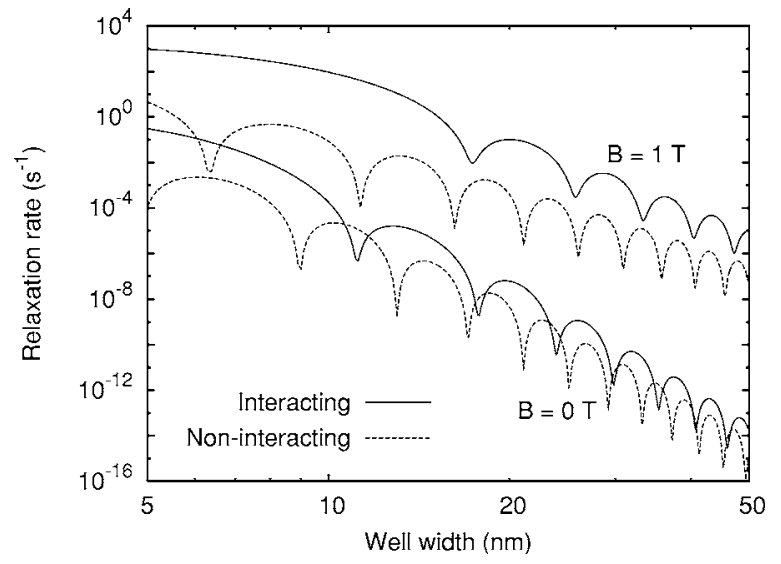

FIG. 2. Comparison of spin relaxation rates for interacting and noninteracting systems.

given of why the integral itself decreases by as much as two orders of magnitude whenever $F_{\perp}$ has a zero. The physical reason is that most phonons are emitted nearly perpendicular to the plane of the dot. This is a consequence of the form of $F_{\|}^{2}$. Each single particle factor, $\left\langle\alpha\left|\exp \left(-i \mathbf{q}_{\|} \cdot \mathbf{r}\right)\right| \beta\right\rangle$ in Eq. (2) has the form $\exp \left(-\lambda^{2} q_{\|}^{2} / 2\right) p\left(q_{\|}\right)$, where $p$ is a polynomial, $\lambda^{2}=\hbar / 2 m^{*} \Omega, \Omega^{2}=\omega_{0}^{2}+\omega_{c}^{2} / 4, \hbar \omega_{0}$ is the confinement energy, and $\omega_{c}$ is the cyclotron frequency. Hence, $F_{\|}^{2}$ has the form $\exp \left(-\lambda^{2} q_{0}^{2} \sin ^{2} \theta\right) P\left(\lambda q_{0} \sin \theta\right)$, where $P$ is another polynomial. For $B \lesssim 2 \mathrm{~T}, q_{0} \lambda$ is typically $>1$ and the exponential factor makes $F_{\|}^{2}$ small unless $\theta$ is also small. Hence, most of the weight in the integral in Eq. (1) comes from the small $\theta$ range where the exponential is large. A rate minimum occurs when this $\theta$ range contains a zero of $F_{\perp}$. The zeros are smeared out because $F_{\perp}$ appears under the integral in Eq. (1) but the smearing is small because of the small $\theta$ range, so the rate reduction near a zero is significant, about two orders of magnitude.

The inset in Fig. 1 illustrates the importance of the perpendicular form factor for the magnitude of the relaxation rate. The solid lines show rates for $B=0 \mathrm{~T}$ and $B=1 \mathrm{~T}$ for the finite barrier case and these rates are identical to those shown in the main part of the figure. The dashed lines show rates calculated with the same parameters but with the finite barrier replaced by an infinite one. The infinite barrier model has been used widely as an idealized dot model (Refs. 9 and 16 , for example) but it is clear that the rates are overestimated in this model, typically by around two orders of magnitude and by about 4 orders of magnitude at worst. The reason is that $\chi(z)$ is broader in the finite barrier case. This reduces $\left\langle p_{z}^{2}\right\rangle$ and has a dramatic effect on the rates.

Figure 2 shows the effect of the interaction between the electrons. The two solid lines are identical to the rates shown in the main part of Fig. 1 and include the effect of the interaction. The dashed lines show the results of repeating the calculations with the interaction excluded. The interaction affects $\Delta E$ and $F_{\|}^{2}$ and this increases the rates by 1 to 3 orders of magnitude. The effect on $\Delta E$ is a decrease by a factor of about 2. This shifts the minima and increases the product $C\left(q_{0}\right) F_{\perp}^{2} \propto 1 / q_{0}^{3}$. The interaction affects $F_{\|}^{2}$ by a factor of 1.5-4 and the effect can be an increase or a decrease,

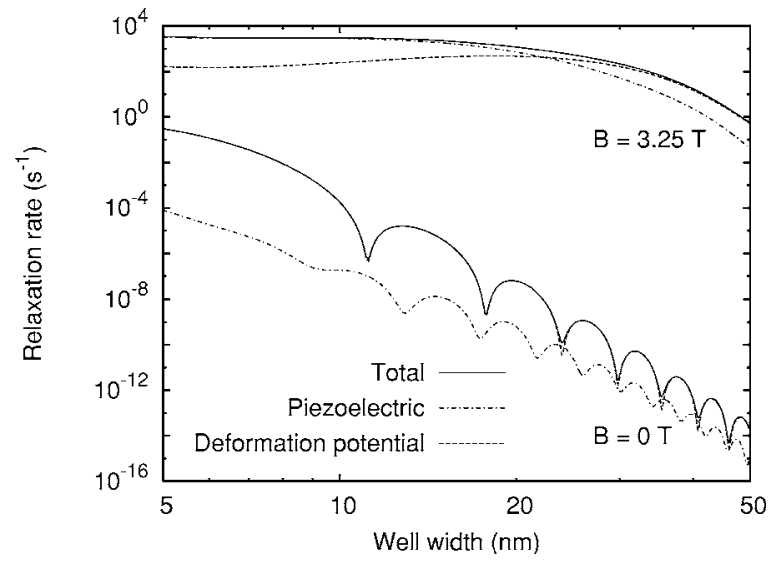

FIG. 3. Comparison of rates of spin relaxation via deformation potential and piezoelectric scattering.

depending on $B$. However, the net effect of the interaction for most values of $w$ is to increase the rates.

The effect of different electron-phonon interaction mechanisms is illustrated in Fig. 3. The figure shows the total rate of relaxation via the deformation potential, longitudinal piezoelectric and transverse piezoelectric interactions together with the deformation potential and total piezoelectric contributions. The figure illustrates the typical behavior in the regimes of large and small $\Delta E$. The different behavior results from the factor $C\left(q_{0}\right)$ in Eq. (1). The $q_{0}^{3}$ factor in the deformation potential case ensures that the deformation potential interaction dominates when $\Delta E$ is large and the curves for $B=0 \mathrm{~T}$ illustrate this; the total and deformation potential rates are indistinguishable except close to the rate minima. The curves for $B=3.25 \mathrm{~T}$ illustrate the opposite regime. This magnetic field is close to the singlet-triplet transition so $\Delta E$ is small throughout the entire $w$ range. However, the singlettriplet transition moves to higher field as $w$ increases because the increasing electron separation weakens the effect of the interaction. Hence, $\Delta E$ increases slowly with $w$. Consequently, the piezoelectric interaction is dominant in the small $w$ regime where $\Delta E$ is smallest and a crossover to a deformation potential dominated regime occurs at $w \sim 22 \mathrm{~nm}$. The piezoelectric interaction was found to dominate in single electron relaxation from Zeeman sublevels in a GaAs dot with infinite barrier confinement, ${ }^{9}$ but both piezoelectric and deformation potential dominated regimes occur in the present triplet-singlet case because of the relatively large $\Delta E$ variation that occurs when $w$ and $B$ are varied.

The magnetic field dependence of the total rate, including all deformation potential and piezoelectric contributions, is shown in Fig. 4. Below the singlet-triplet transition ( $B$ $\simeq 3.8 \mathrm{~T}$ for $w=12 \mathrm{~nm}$ and $B \simeq 4.3 \mathrm{~T}$ for $w=24 \mathrm{~nm}$ ) there are oscillations similar to those in Fig. 1. They are very pronounced for $w=24 \mathrm{~nm}$ because the $q_{\perp}$ distance between zeros decreases with $w$. This can be seen by considering the large $w$ approximation to $F_{\perp}$, Eq. (4). Within this approximation the zeros occur when $(1-\varepsilon) q_{0} w \cos \theta / 2=n \pi$. And since $\varepsilon$ is small $(<0.15$ when $w \gtrsim 24 \mathrm{~nm})$ and $\cos \theta \sim 1$, the difference between $q_{0}$ at successive zeros is approximately $2 \pi / w$. This decreases with $w$ so the number of zeros, hence rate minima, increases, as illustrated in the 


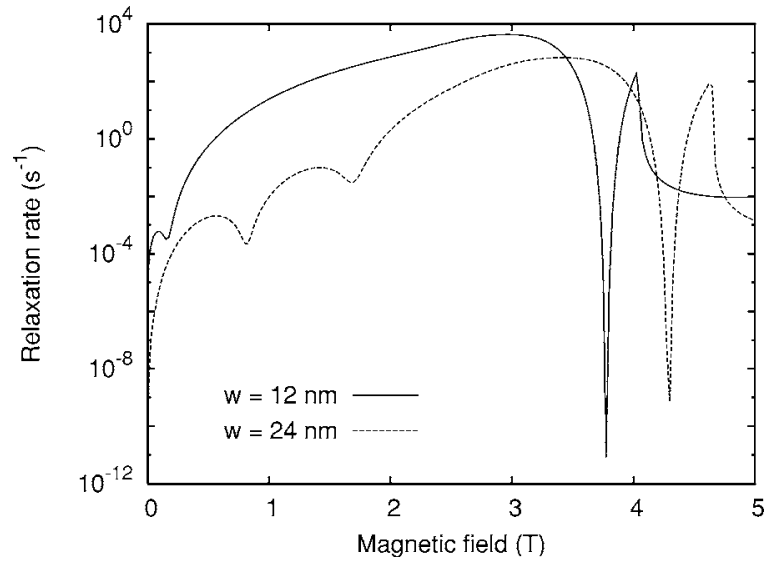

FIG. 4. Spin relaxation rate as a function of $B$.

figure. Oscillations similar to those in Fig. 4 have been reported for energy relaxation in double dots ${ }^{19}$ and relaxation between Zeeman sub-levels ${ }^{9}$ but the present analysis shows that the effect should occur in any system where $F_{\perp}$ has zeros, provided that $\Delta E$ is of appropriate magnitude. The results in Fig. 4 suggest that a wide well is the best choice for experimental observation of the rate oscillations. Beyond the singlet-triplet transition there is a peak in the rate which is associated with the anticrossing shown in the inset of the top frame of Fig. 1 and at the transition there is deep minimum. This structure would change in the presence of Rashba coupling. Strong Rashba coupling is known to cause a rate peak at the singlet-triplet transition ${ }^{10}$ in lateral dots but the effect is likely to be weaker in the device considered here.

\section{CONCLUSION}

The rate of triplet-singlet relaxation via spin mixing has been studied with a realistic two-electron dot model. The rate oscillates with $w$ in an envelope that scales approximately like $1 / w^{18}$ when $B=0 \mathrm{~T}$ and $1 / w^{10}$ when $B \neq 0$. This means a small increase in $w$ can put a dot into a regime where the linear Dresselhaus spin mixing mechanism is suppressed dramatically. Spin relaxation in this regime should be dominated by other mechanisms, such as the third order Dresselhaus mechanism, ${ }^{4}$ coupling to the phonon strain field, ${ }^{4}$ or the hyperfine interaction. ${ }^{21}$ All these mechanisms involve interaction with phonons but in each case $F_{\|}$does not depend significantly on $w$. So the rates scale like $1 / w^{6}$ in the first two cases and $1 / w^{5}$ in the third, hence they must become dominant when $w$ is sufficiently large. Although detailed investigations for the device considered here have not yet been done, the available results ${ }^{4,21}$ suggest that the rate for each mechanism greatly exceeds the rate for the linear Dresselhaus mechanism in the large $w$ regime. It is also possible that Rashba coupling could affect the rate but this effect is likely to be small in the nearly symmetric device considered here. Experiments on the $w$ or $B$ dependence of the rate could be used to test the present theory but, because of the very high sensitivity of the rate to $w$ and $\Delta E$, this would require an extremely well characterized dot.

While this work was being prepared for publication, a preprint was received from Pietiläinen and Chakraborty. ${ }^{22} \mathrm{As}$ part of a study of magneto-optics, they have developed a method to diagonalize the Hamiltonian with Rashba spinorbit coupling which is similar to the method developed here for the Dresselhaus case.

We have recently received a preprint from Climente et $a l .{ }^{23}$ who have studied triplet-singlet relaxation in two and four electron parabolic dots. Their results for two electrons are consistent with those presented here.

\section{ACKNOWLEDGMENTS}

We thank M. Roy for critically reading the manuscript and making useful comments. This work was supported by the UK Engineering and Physical Sciences Research Council.
*Present address: Gooch and Housego PLC, The Old Magistrates Court, Ilminster, Somerset TA19 0AB, United Kingdom.

${ }^{1}$ T. Fujisawa, D. G. Austing, Y. Tokura, Y. Hirayama, and S. Tarucha, Nature (London) 419, 278 (2002).

${ }^{2}$ R. Hanson, B. Witkamp, L. M. K. Vandersypen, L. H. Willems van Beveren, J. M. Elzerman, and L. P. Kouwenhoven, Phys. Rev. Lett. 91, 196802 (2003).

${ }^{3}$ J. M. Elzerman, R. Hanson, L. H. Willems van Beveren, B. Witkamp, L. M. K. Vandersypen, and L. P. Kouwenhoven, Nature (London) 430, 431 (2004).

${ }^{4}$ A. V. Khaetskii and Y. V. Nazarov, Phys. Rev. B 61, 12639 (2000).

${ }^{5}$ S. Dickmann and P. Hawrylak, JETP Lett. 77, 30 (2003).

${ }^{6}$ M. Florescu, S. Dickman, M. Ciorga, A. Sachrajda, and P. Hawrylak, Physica E (Amsterdam) 22, 414 (2004).

${ }^{7}$ A. V. Khaetskii and Y. V. Nazarov, Phys. Rev. B 64, 125316 (2001).

${ }^{8}$ V. I. Falko, B. L. Altshuler, and O. Tsyplyatev, Phys. Rev. Lett.
95, 076603 (2005).

${ }^{9}$ C. F. Destefani and S. E. Ulloa, Phys. Rev. B 72, 115326 (2005).

${ }^{10}$ M. Florescu and P. Hawrylak, Phys. Rev. B 73, 045304 (2006).

${ }^{11}$ Y. Nishi, P. A. Maksym, D. G. Austing, T. Hatano, L. P. Kouwenhoven, H. Aoki, and S. Tarucha, Phys. Rev. B 74, 033306 (2006).

${ }^{12}$ P. A. Maksym, Y. Nishi, D. G. Austing, T. Hatano, L. P. Kouwenhoven, H. Aoki, and S. Tarucha (unpublished).

${ }^{13}$ L. P. Kouwenhoven, D. G. Austing, and S. Tarucha, Rep. Prog. Phys. 64, 701 (2001)

${ }^{14}$ T. Hassenkam, S. Pedersen, K. Baklanov, A. Kristensen, C. B. Sorensen, P. E. Lindelof, F. G. Pikus, and G. E. Pikus, Phys. Rev. B 55, 9298 (1997).

${ }^{15}$ E. A. de Andrada e Silva, G. C. La Rocca, and F. Bassani, Phys. Rev. B 55, 16293 (1997).

${ }^{16}$ M. Valín-Rodríguez, A. Puente, and L. Serra, Phys. Rev. B 66, 045317 (2002).

${ }^{17}$ C. F. Destefani, S. E. Ulloa, and G. E. Marques, Phys. Rev. B 70, 
205315 (2004).

${ }^{18}$ P. Lucignano, B. Jouault, A. Tagliacozzo, and B. L. Altshuler, Phys. Rev. B 71, 121310(R) (2005).

${ }^{19}$ A. Bertoni, M. Rontani, G. Goldoni, F. Troiani, and E. Molinari, Appl. Phys. Lett. 85, 4729 (2004).

${ }^{20}$ K. Kalna, S. Roy, A. Asenov, K. Elgaid, and I. Thayne, SolidState Electron. 46, 631 (2002).
${ }^{21}$ S. I. Erlingsson, Y. V. Nazarov, and V. I. Falko, Phys. Rev. B 64, 195306 (2001).

${ }^{22}$ P. Pietiläinen and T. Chakraborty, Phys. Rev. B 73, 155315 (2006).

${ }^{23}$ J. I. Climente, A. Bertoni, G. Goldoni, M. Rontani, and E. Molinari (unpublished) 